\title{
Covid-19: Repercusiones en la educación universitaria
}

\author{
Covid-19: Impact on University Education
}

Covid-19: Repercussões na educação universitária

\section{ARTÍCULO GENERAL}

$\begin{array}{ccc}\text { Walter Humberto } & \text { Hugo Eliazar } & \text { Juan Henrry } \\ \text { Gallegos Elias } & \text { Maldonado Espinoza } & \text { Añanca Rojas } \\ \text { waltergallegos@gmail.com } & \text { hugoeliazar@gmail.com } & \text { juanhenry@gmail.com } \\ \text { https://orcid.org/0000-0002-0187-3474 } & \underline{\text { https://orcid.org/0000-0003-0834-2139 }} & \underline{\text { https://orcid.org/0000-0002-4628-3873 }} \\ \text { mestigador Independiente, Lima - Perú } & \text { Investigador Independiente, Lima - Perú } & \text { Investigador Independiente, Lima - Perú }\end{array}$

Recibido 07 de Junio 2021 | Arbitrado y aceptado 25 de Agosto 2021 | Publicado en 04 Diciembre 2021

\section{RESUMEN}

Desde el comienzo de la pandemia se han generado cambios muy drásticos en la vida de todas las personas, impactando de manera desfavorable en los estudiantes universitarios en todo el mundo. La pandemia ocasionó la suspensión de la enseñanza presencial, por lo que se buscaron alternativas para continuar el proceso docente-educativo aún en condiciones de restricciones, aislamiento social, entre otros inconvenientes. La vida de los estudiantes universitarios de igual manera ha cambiado radicalmente debido a la coyuntura actual y a las reformas que ésta ha generado. Por este motivo, muchas de ellas han cambiado su modalidad a netamente virtual. Según este contexto, la presente investigación busca analizar las repercusiones en los estudiantes en el marco de la cuarentena por COVID-19.

Palabras claves: Educación virtual; COVID-19; Estudiante universitario.

\section{ABSTRACT}

Since the beginning of the pandemic, very drastic changes have been generated in the lives of all people, negatively impacting university students around the world. The pandemic caused the suspension of face-to-face teaching, so alternatives were sought to continue the teaching-educational process even under conditions of restrictions, social isolation, among other inconveniences. The life of university students has also radically changed due to the current situation and the reforms that it has generated. For this reason, many of them have changed their modality to purely virtual. In this context, this research seeks to analyze the repercussions on students in the framework of the COVID-19 quarantine.

Keywords: Virtual education; COVID19; College student.

\section{RESUMO}

Desde o início da pandemia, mudanças muito drásticas foram geradas na vida de todas as pessoas, impactando negativamente estudantes universitários em todo o mundo. A pandemia ocasionou a suspensão do ensino presencial, buscando alternativas para dar continuidade ao processo ensino-educativo mesmo em condições de restrições, isolamento social, entre outros inconvenientes. A vida dos universitários também mudou radicalmente devido à situação atual e às reformas que ela gerou. Por esse motivo, muitos deles mudaram sua modalidade para puramente virtual. Nesse contexto, esta pesquisa busca analisar as repercussões sobre os alunos no âmbito da quarentena do COVID-19.

Palavras-chave: Educação virtual; COVID-19; Estudante universitário. 


\section{Introducción}

Desde los inicios, la humanidad ha tenido que ir pasando por cambios abruptos y repentinos que han sido causa del replanteamiento de sus modelos de vida. La coyuntura actual, se ha marcado por la existencia de una nueva pandemia mundial, que como resultado ha alterado en muchas formas la vida cotidiana de todos peruanos del siglo XXI, siendo uno de los más ampliamente afectados, sin duda, la educación universitaria. De esta manera, la pandemia del COVID-19 ha obligado a los centros de educación universitaria a postergar las clases dictadas presencialmente y a reemplazarlas por ciclos netamente virtuales (La República 2020, párr. 1). Circunstancias como esta parece revalidar lo mencionado por Chiecher, Donolo y Rinaudo (2005, p. 3) cuando resaltan la importancia de preparar a los estudiantes universitarios a emplear nuevas modalidades para recibir sus clases, en especial en el uso de la tecnología. Mucho se ha escrito sobre la creciente educación virtual y sus ventajas en la educación superior. Sin embargo, la gran mayoría de las universidades peruanas no se encontraban preparadas para afrontar el desafío de una educación no presencial. En especial cuando ella fue fruto de una adaptación forzosa. Ella no solo nos recuerda las carencias del sistema de educación superior.

Diversos son los autores que han estudiado y evaluado diversas medidas para obtener una buena experiencia en aulas virtuales. Autores como Area y Adell (2009, p. 8) se centran, en especial, en la comunicación e interacción entre los agentes involucrados en el elearning, porque en esta se encuentra el éxito de la educación netamente virtual. Los autores antes ya mencionados señalan que las problemáticas actuales de las clases virtuales giran en torno a la calidad de la educación impartida de forma virtual. Por su parte, Lara (2001) enfatiza el carácter diverso de las estrategias didácticas en un entorno virtual y clasifica los recursos en sincrónicos y asincrónicos. Además, propone que se deben utilizar lo mejor de ambos recursos para obtener una mayor productividad en la educación no presencial. Chumpitaz (2002, p. 84) estudia tanto al docente como al alumno virtual y ofrece interesantes alcances sobre las funciones y características de cada uno de estos para una experiencia virtual fructífera. Sin embargo, a diferencia de los autores antes citados, es Gagliardi (2020,

p. 4) quien se cuestiona sobre algunos de los problemas que los estudiantes podrían presentar en un aula virtual. Los factores como la disponibilidad de recursos tecnológicos y la calidad de estos son recogidos por la autora como conceptos que se tienen que tomar 
en cuenta para el buen desenvolvimiento de los estudiantes en el nuevo reto de una virtualización de las aulas inesperada y difícil.

Estudios como el presente articulo ayudan a comprender y mejorar las condiciones que actualmente los universitarios enfrentan en la cuarentena. Además, podrían ser utilizadas para formar parte de base de investigaciones futuras para una implementación definitiva de la educación no presencial en los centros de educación universitaria.

\section{Metodología}

La metodología que se utilizó consta de una búsqueda de información a base de papers, revistas, análisis de aplicaciones actuales y páginas web especializadas para entender su funcionamiento, el nivel de desarrollo obtenido en la actualidad y cuáles son sus principales aplicaciones. Además, se han empleado análisis de modelos estratégicos acordes para analizar el atractivo de las industrias en la adopción de la tecnología y así determinar a través de modelos de análisis de negocio cuáles son las industrias en que tendrá mayor incidencia.

La búsqueda de la información para la revisión de literatura se hizo consultando las siguientes bases de datos como son, Scopus, Proquest y Google Académico para encontrar revistas del más alto nivel científico. Asimismo, cabe mencionar que para la realización de lo mencionado se emplearon también palabras clave como educación virtual; COVID-19; Estudiante universitario.

Cada uno de estos descriptores se combinaron entre sí durante la búsqueda utilizando los operadores boléanos "and" y "or". Además, algunos de los criterios o filtros que se utilizaron para hacer muchos más específica la búsqueda y ser más precisos con los documentos encontrados en las diferentes bases de datos consultadas fueron: que sean artículos de revistas, y la antigüedad de la publicación que no sea mayor a 5 años.

Toda la información previa relacionada a los filtros aplicados y las especificaciones sirvieron como criterios de inclusión, es decir que sean artículos publicados en revistas científicas, que no excedan los cinco años de antigüedad, que la temática guarde relación con las variables de creatividad artística y tiempos de pandemia. Se excluyeron, en tanto, todos los documentos que no respetaran lo postulado así como aquellos que estaban incompletos o que tenían enlaces averiados. 


\section{Resultados y discusión}

\subsection{Nociones básicas}

\section{Educación virtual no presencial}

El método de educación no presencial, cuya principal característica es la carencia del factor de temporalidad, no es algo nuevo en el mundo. La aparición de esta se remonta años atrás, después de la aparición de educación por correspondencia, radio y televisión. La educación a distancia, naturalmente, es más susceptible a la incorporación de las nuevas tecnologías que su predecesora, la educación presencial (Ruiz y Domínguez, 2007).

El método de enseñanza presencial se caracteriza por tener al alumno y al profesor en el mismo espacio temporal y físico (Cursi, 2003). A diferencia de la enseñanza clásico, en la que el instructor es la primordial fuente de información, en la modalidad a distancia el alumno se convierte en el adquisidor activo del entendimiento, en lo que el maestro consigue un papel de orientador.

La enseñanza virtual se ha convertido en una modalidad de enseñanza no presencial que se asocia a la implementación de tecnologías de la información y la comunicación para la construcción de la enseñanza-aprendizaje. Frecuenta ser percibida como un sistema de aprendizaje innovador, con base en la red en línea, que ha roto con las brechas de tiempo y de espacio que suponen la enseñanza presencial (Garrison y Anderson, citado por Azuaje, 2012, Llopiz, Andreu, Gónzalez, Alberca, Fuster-Guillén y Palacios-Garay, 2020). Siguiendo el cumplimiento de lo expresado por los autores, la enseñanza virtual coopera a los planteado por Thomas y Loxley (2007) ambos manifiestan que una enseñanza de carácter inclusivo involucra la democratización de la enseñanza. No obstante, el entorno de postergación de clases presenciales obligó a las instituciones de enseñanza mayor a llevar a cabo la enseñanza virtual de una forma acelerada. Aun cuando ciertos universitarios se han observado beneficiados con el acortamiento de las brechas mencionadas por los autores, son bastantes los dañados por otro tipo de brechas, de naturaleza social y digital. Chaves (2017) plantea que, en la llamada sociedad del entendimiento, la enseñanza clásica aún muestra deficiencias en lo en cuanto a la utilización de TIC y al alcance y calidad de dichos. Postulados como el anterior son exactos en tiempos de enfermedad pandémica, donde dichas deficiencias se acentúan.

\section{Rol profesor - estudiante en la educación virtual}

Si bien la evaluación de que la sociedad propició el principio de las clases virtuales, la enfermedad pandémica del coronavirus ha obligado tanto a los maestros como a los 
alumnos a ajustarse a una enseñanza 100\% virtualizada. Ello, paralelamente, ha implicado un cambio en las tácticas de aprendizaje y educación de dichos, además de una redefinición de sus papeles.

En la situación de los docentes, varios autores ya han reconocido, e inclusive usado, un nuevo vocabulario que sustituye el término "profesor" por "tutor" para conceptualizar al profesor en salas virtuales (Chaupart, Corredor y Marín, 1998, Silva, 2010). El concepto involucra una transformación en la metodología y papel que comunmente desempeñan los docentes. En este cambio, el instructor deja de ser la primordial o exclusiva fuente de información verídica, puesto que los alumnos poseen actualmente infinidad de fuentes de esta, para ser un orientador del alumno, quien construye sus conocimientos en grupo con este (Silva, 2010, Bolívar y Dávila, 2016). Ello involucra, como estamos de consenso, un conveniente entendimiento y funcionamiento de las TIC y demás artefactos tecnológicos para una buena orientación de los alumnos (Coll, 2008, Gros y Silva, 2005). Alonso y Blázquez (2016) resumen las funcionalidades del maestro virtual en funcionalidad profesor, elaborando materiales didácticos, funcionalidad de orientación, desempeñando tácticas fundamentadas en la empatía y comprensión que posibiliten el aprendizaje, y funcionalidad técnica, conociendo los conceptos básicos para la implementación de TIC. Considerando el entorno presente que por sí mismo perjudica la salud psicológica de los individuos, es fundamental que el maestro enseñe teniendo presente la capacidad de orientación mencionada por los autores, con empatía y fervor a los universitarios. Ello, puesto que, ahora más que jamás, es imperante una enseñanza a partir de la ética de la vida que forme posturas causantes para el desarrollo de una sociedad más justa (Novoa y Pirela, 2020).

Así mismo, el término para conceptualizar a un estudiante en salas virtuales cambia, transformándose de "estudiante" a "aprendiz", con todo lo cual ello connota (Chaupart, Corredor y Marín, 1998). El "aprendiz" se diferencia del "estudiante" en que el primero es el prota en el nuevo modelo educativo. De esta forma, los estudiantes dejan de ser básicas receptores de información para ser los constructores de sus propios conceptos, orientados por el tutor (Chaupart, Corredor y Marín, 1998). Lo previamente dicho involucra una optimización en la funcionalidad de organización y de la autodisciplina en los alumnos, gracias a la independencia que dichos disponen (Rugeles, Mora y Metaute, 2015). Asimismo, se incrementa la necesidad de un alumno capaz de lidiar con la 
incertidumbre y ambigüedad que implica el tener distintas fuentes de entendimiento (Bautista, Borges y Forés, 2006).

Con relación a la deserción de estudiantes virtuales, La Madriz (2016, p. 23) asegura lo próximo:

Si bien es cierto que, la utilización de los espacios virtuales de aprendizaje permite más grande ingreso a la enseñanza y optimización las oportunidades de obtener información, estas no sustituyen todos los recursos pedagógicos clásicos, sino que precisamente incrementan y diversifican las modalidades del aprendizaje, lo cual deja en prueba que es un desafío para las universidades, apartamentos o cátedras que oferten este sistema virtual de enseñanza, reducir los índices de deserción de estos ámbitos, reto que pasa por tener en cuenta los papeles de los competidores en el proceso, las propuestas y tácticas de educación, los medios u objetos de educación, así como la composición y diseño de la plata- forma educativa, en funcionalidad a que se adapten a las necesidades recientes y futuras de los usuarios.

Si tomamos presente la coyuntura en la que se empezaron las clases virtuales y los efectos que esta tendría a futuro, además de la velocidad con la que se empezó el proceso de habituación de estas, los desafíos a los que el creador hace referencia, que podrían terminar en deserción, se intensifican y se expanden.

IMPLICACIONES DE LA RESPUESTAS A LA PANDEMIA PARA LAS INSTITUCIONES Y SISTEMAS

\section{Retos inmediatos a confrontar:}

- Disrupción institucional a gran escala.

- Limitada asistencia a estudiantes y docentes.

- Desplazamiento masivo de estudiantes.

- Mantenimiento de operaciones instructivas, incluyendo los trabajos de las asignaturas, exámenes, asignación de calificaciones, modificación de las modalidades de evaluación.

- Mantener o cerrar operaciones de investigación que incluye laboratorios e instalaciones en los campus, y de colaboraciones externas.

- Cierre de movilización nacional o internacional. 
- Implicaciones de equidad -académicas, sociales, financieras, físicas- para estudiantes de bajos ingresos y en riesgo (potencialmente aquellos con vulnerabilidades de salud si tienen COVID-19)

\section{Retos a largo plazo}

- Incremento de la desigualdad e inequidad en el acceso y retención estudiantil, como estudiantes con restricciones financieras y otras situaciones (obligaciones familiares, cambios en circunstancias personales, falta de apoyo informático, o campus cerrados y desmantelados).

- Reducción de apoyo privado (se da en algunas universidades privadas que trabajan con empresas, por ejemplo).

- Cierre permanente de programas, y aún instituciones, con reducción de recursos académicos y administrativos, y desempleo.

- Movimiento permanente hacia plataformas remotas u "online", que requiere apoyo para que esto sea efectivo.

- Impactos socio-emocionales en estudiantes (y profesores) por esta enseñanazaapendizaje remota, dando mucha atención al desarrollo de habilidades interpersonales en contextos donde falta experiencias interpersonales.

- Pérdida de las contribuciones de la educación superior a las comunidades cívicas y a la cultura.

- Pérdida de investigaciones.

\section{ACCIONES Y CONSIDERACIONES}

Algunas consideraciones y acciones institucionales que se pueden seguir al mismo tiempo que se trabaja hacia la adaptación a los cambios en la educación terciaria.

\section{Acciones inmediatas /Consideraciones:}

\section{Cierres institucionales:}

- Establecer protocolos transparentes, a tiempo y con una comunicación consistente con la comunidad (estudiantes, docentes, trabajadores, asociados) en relación a las necesidades de cierre y los pasos que se van a tomar para asegurar una transición adecuada.

- Asegurar a la comunidad acceso a la información y a las decisiones tomadas. 
- Delegar a las autoridades académicas los cierres en sus respectivas especialidades (Escuelas Profesionales), ya que ellos conocen todos los procedimientos y apoyar en la suspensión de clases e investigación, si procede.

- Si se va a continuar con las labores, identificar las necesidades e infraestructura (energía, banda ancha) y equipamiento (laptops, "tablets", plataformas) para reforzar el trabajo, asegurando también el financiamiento.

- Evaluar un plan de asuntos de seguridad para mantener edificios vacíos con equipos y materiales costosos.

\section{Oferta de los cursos:}

- Entrenar a instructores para la enseñanza remota y la evaluación de esta nueva modalidad.

- Buscar o adaptar plataformas para enseñanza "online" o remota, pensando en que los cursos tradicionales persona-persona no son factibles.

- Evaluar la capacidad de los estudiantes para integrarse al aprendizaje remoto - equipos, responsabilidades familiares, entorno hogareño- para entender cuan realista es para los estudiantes adaptarse a estas nuevas estrategias virtuales.

- Proveer de equipos a estudiantes y profesores cuando sea posible (lo está haciendo el Ministerio de Educación para dar "tablets" a los estudiantes).

- Políticas de evaluación más flexibles para calificar a los estudiantes o para pasar los cursos.

- Evaluar profesores y cursos.

- Mejorar los programas de tutoría para las personas de contacto para estudiantes que no se adaptan o tienen otros problemas.

\section{Investigación (cuando sea relevante):}

- Apoyar a los investigadores para esta transición, donde sea posible, llevar a cabo investigaciones con plataformas remotas. 
- Mantener equipos y tecnologías para permitir tener nivel de continuidad de investigaciones.

- Limitar la exposición de los investigadores a riesgos derivados de la pandemia.

- Mantener una lista de espacios esenciales y materiales de investigación para una rápida respuesta en caso de una emergencia.

\section{Prepararse para el próximo año académico:}

- Prepararse para un nuevo escenario y enfocarse en reasumir la operación normal de las tareas universitarias.

- Evaluar permanentemente los efectos de la educación remota y adaptarse y resolver retos.

\section{Mantenimiento de operaciones organizacionales:}

- Suspender razonablemente pagos y gastos no esenciales.

- Establecer reuniones, regulares y consistentes, de las autoridades y funcionarios para asegurar su involucramiento en la gestión de la institución y la evolución integral de la misma bajo estas circunstancias extraordinarias.

- Mantener una ligazón permanente con el staff operativo y confrontar rápidamente retos operativos que se presenten a todos los niveles administrativos y académicos de la organización.

\section{CONSIDERACIONES A LARGO PLAZO PARA LOS GOBIERNOS:}

- El desempleo de los profesionales va a crecer, debido a la contracción económica y la desconfianza del mercado laboral en la calidad del aprendizaje remoto. Hay que considerar la expansión de cursos cortos y programas de certificación para proveer opciones de aprendizaje rápido de habilidades a los estudiantes terciarios.

- Revisar las regulaciones y barreras burocráticas para adecuarse a las nuevas realidades de la enseñanza, aprendizaje e investigación.

- Considerar dedicar programas de apoyo (financieros, logísticos, pedagògicos) para estudinates vulnerables que no pueden acceder a estas nuevas modalidades). 


\section{HABILITACIÒN DE EDUCACIÒN “ONLINE" Y A DISTANCIA PARA LA EDUCACIÒN TERCIARIA}

\section{Convertir la oferta tradicional a una online:}

En respuesta a la crisis del COVID-19, muchos países e instituciones de educación terciaria han sido capaces de transformar sus operaciones "online" de manera relativamente rápida. Una razón para que algunos se hayan adaptado más rápido es que ya habían hecho inversiones en conectividad digital hace algunos años o décadas.

\section{ACCIONES PARA LOS LİDERES EN EL CORTO Y MEDIANO PLAZO}

- Desarrollar planes para el corto, mediano y largo plazos. El corto plazo se enfocará en una respuesta de emergencia para mantener a los estudiantes aprendiendo. El mediano y largo plazo será para preparar a las universidades a reabrirse, construyendo resiliencia y transformación.

- Utilizar los recursos y las tecnologías más utilizadas localmente (en cada país), como, por ejemplo, videoconferencias y plataformas de enseñanza aprendizaje ya conocidas.

- Enfocarse en contenidos existentes- más que en desarrollar nuevos contenidos, ya que estos requieren tiempo y "expertise"- y alinearlos a los currículos existentes.

- Ofrecer orientación regular y permanente a estudiantes y profesores es fundamental: disponer de comunicaciones regulares, dar consejos proactivos e implementar un escritorio de ayuda ("help desk").

\section{SOSTENER NUESTROS VALORES DURANTE LA CRISIS}

Las comunidades de educación superior importan en gran medida en esta etapa de pandemia y crisis. Son las máquinas de producción de conocimientos, descubrimientos, innovación, habilidades para el desarrollo, preservación cultural y progreso nacional (y diría de la producción de profesionales e investigadores).

Pero para tener comunidades saludables hay que tener valores de base y nucleares: acceso equitativo, rendición de cuentas, libertad académica, autonomía institucional y responsabilidad social. 
En la carrera para responder a los cambios inmediatos -eliminando los caminos del contagio de la pandemia, cerrando instituciones y haciendo muchos esfuerzos en la enseñanza, el aprendizaje y la investigación- es fácil enfocarse en las ofertas y respuestas que se tienen que dar. ¿Cómo enseñamos a aquellos que quieren aprender? ¿Cómo apoyamos para que continúe la investigación? Esto es lo lógico e importante como primera respuesta. Es imperativo, sin embargo, pensar más allá de las necesidades urgentes y de sobrevivencia, y mantener un ojo en los valores de la educación terciaria, para no dejarse abatir y continuar con la misión de la educación superior.

Los aprendizajes a distancia y "online" han forzado a una adaptación masiva sobre cómo distribuir información y cómo los estudiantes deben aprender. Los estudiantes con retos de aprendizaje se dejan atrás, así como los estudiantes con discapacidades. Las instituciones lejos de los centros urbanos se dejan de lado. Esto ya era verdad antes de la pandemia, pero lo que sucede con la pandemia es que se exacerba la velocidad a la cual las disparidades afectan la persistencia estudiantil y la sobrevivencia de las instituciones.

Los líderes educativos deben ver y producir evidencia de las ciencias educativas y de las innovaciones tecnológicas, para asegurar que estos cambios de enseñanza “online" y plataformas de distribución de información realmente son una promesa para el desarrollo de aprendizajes y habilidades. Se debe analizar la eficacia y entender mejor lo que funciona y para quién funciona.

Al hacer esto, y mientras lideran sus sistemas educativos en un mundo postcrisis, los que hacen las políticas, y los ejecutores de las mismas, necesitan enfocar sus esfuerzos en las estudiantes más vulnerables, asegurando que las soluciones de enseñanza y aprendizaje, las ayudas tecnológicas, la inversión en infraestructura, se orienten hacia aquellos estudiantes involucrados y conectados y apoyar sus procesos y resultados.

\section{Conclusiones}

Previo a la enfermedad pandémica del coronavirus, el planeta ya estaba experimentando una crisis educativa. Y la crisis no estaba distribuida igualmente: esos que viven en situación de mayor desventaja poseen un peor ingreso a la escolaridad, más grandes tasas de deserción estudiantil y más grandes déficits de aprendizaje. 
La enfermedad pandémica del coronavirus ya ha provocado impactos profundos en la enseñanza, a partir del instante en que se cerraron las escuelas de todo el mundo en la más grande conmoción que hayamos experimentado de forma simultánea en nuestras propias vidas. El mal va a ser todavía más severo mientras la emergencia sanitaria se traduzca en una fuerte recesión universal. El efecto ocasionado por el cierre de escuelas conducirá a una pérdida de aprendizajes, un crecimiento en la deserción estudiantil y una más grande diferencia; el efecto económico exacerbará el mal, al deprimir la oferta y la demanda educativa, a la vez que provocará perjuicios a los domicilios; y todo ello significará un precio a extenso plazo en términos de acumulación de capital humano, prospectos de desarrollo y confort.

No obstante, si las naciones se mueven con velocidad para ayudar la continuidad del aprendizaje, podrían mitigar los perjuicios, por lo menos parcialmente. Y con el planeamiento y las políticas adecuados podrían aprovechar esta crisis como una oportunidad para desarrollar sistemas educativos más inclusivos, eficientes y resilientes. Las políticas que surjan para lograrlo tienen la posibilidad de resumirse en 3 etapas que se sobreponen:

Afrontamiento, Desempeño de la Continuidad, y Agilizar el Aprendizaje.

A lo extenso de la etapa de Afrontamiento muchas naciones implementaron de forma innovadora el aprendizaje a distancia. No obstante, ya que las naciones no se encontraban preparados para un efecto negativo como este, todos permanecen aprendiendo en todo el camino que tienen que intentar de usar diferentes plata- maneras que les permitan llegar a la más grande porción viable de alumnos. Y este es el desafío crítico. Si no se cuenta con políticas explícitas para llegar a los domicilios más vulnerables, solamente las familias de elevados recursos económicos y con buen grado educativo van a ser capaces de confrontar las secuelas.

Una vez que se se encuentre en la etapa de Desempeño de la Continuidad, los sistemas educativos tienen que intentar eludir las reducciones peligrosas (y probablemente irreversibles) respecto a matrículas y para cerrar las brechas de aprendizaje que, posiblemente, se hayan ampliado a lo extenso de el cierre. Los esfuerzos de- ben orientarse a indemnizar la época perdido para eludir impactos permanentes sobre el capital humano de chicos y adolescentes en la actualidad en edad estudiantil. Esto 
necesitará de una secuencia de medidas dirigidas a revertir las pérdidas en los aprendizajes, a partir de evaluaciones mejoradas del salón de clase hasta pedagogías focaliza- provees en la ayuda a grado sistema. Además, se necesitarán recursos fundamentales, a la vez que tienen que protegerse los presupuestos de enseñanza en un rato en que las familias cuentan con menos ingresos accesibles para favorecer la enseñanza en el hogar y las solicitudes del sistema público posiblemente se aumenten.

No obstante, de la mano de la contestación y las in- novaciones originadas por la crisis, viene una enorme posibilidad para desarrollar sistemas educativos más fuertes y equitativos que Anteriormente a lo largo de la etapa de Apresurar el Aprendizaje. Después de la pandemia, el colegio va a ser distinta. varios actores - padres, profesores, medios de comunicación, régimen y otros - habrán cambiado sus opiniones y percepciones acerca del papel que poseen en el proceso de educación. Ejemplificando, los papás comprenderán mejor la necesidad de laborar de forma conjunta con las escuelas para impulsar la enseñanza de sus hijos, en lo que los medios de comunicación comprenderán el crítico papel que todavía poseen la TV y la radio y su inmensa responsabilidad. Habrá una mejor comprensión sobre la separación digital: tanto las diferencias en la entrada al hardware, la conectividad y el programa conveniente, como además acerca del gran déficit de docentes que tengan capacidades digitales. Muchas brechas de equidad habrán sido puestas en prueba, y la necesidad urgente de actuar sobre las mismas quedará más clara. Asimismo, las innovaciones generadas en las etapas de Afrontamiento y Desempeño de la Continuidad habrán demostrado lo cual puede hacerse una vez que las naciones se centran en enfoques más efectivos y equitativos para cerrar las brechas de aprendizaje para todos los chicos. Las comunidades, por consiguiente, poseen una verdadera posibilidad de "reconstruir mejor" y de usar las tácticas más efectivas de recuperación frente a la crisis como base para mejoras a largo plazo.

\section{Referencias}

Azuaje, D. (2012). Educación Presencial, Educación a Distancia, Educación Semipresencial o Mixta y Educación Virtual. Trabajo Especial de Grado de la Especialización en “Telemática e Informática en la Educación a Distancia”.

Bautista, G., Borges, F., y Forés, A. (2006). Didáctica universitaria en entornos virtuales de enseñanza-aprendizaje (Vol. 13). Madrid: Narcea ediciones. 
Bolívar, C. R. y Dávila, A. A. (2016). Propuesta de buenas prácticas de educación virtual en el contexto universitario. Revista de Educación a Distancia, (49). doi: http://dx.doi.org/10.6018/red/49/12

Chaupart, J. M., Corredor, M. V. y Marín Muñoz, G. I. (1998). El tutor, el estudiante y su nuevo rol. Universidad de Guadalajara. En Desarrollo de ambientes de aprendizaje en educa- ción a distancia (pp. 97-110). Guadalajara: Universidad de Guadalajara. Recuperado de

Chaupart, J. M., Corredor, M. V. y Marín Muñoz, G. I. (1998). El tutor, el estudiante y su nuevo rol. Universidad de Guadalajara. En Desarrollo de ambientes de aprendizaje en educa- ción a distancia (pp. 97-110). Guadalajara: Universidad de Guadalajara. Recuperado de http://www.isiberico.com/documentos/tutorestudianteynuevorol.PDF Chaves, A. (2017). La educación a distancia como respuesta a las necesidades educativas del siglo XXI. Academia y Virtualidad, 10(1), 23-41. doi: http://dx.doi.org/10.18359/ravi.2241

Chiecher, A., Donolo, D., y Rinaudo, M. (2005). Percepciones del aprendizaje en contextos presenciales y virtuales. La perspectiva de alumnos universitarios. Revista de educación a distancia, (13), 1-10. Recuperado de https://revistas.um.es/red/article/view/24401

Chumpitaz, L. (2002). Aprendiendo en los entornos virtuales. Revista Educación, 11(21), $77-$

\section{Recuperado de}

http://revistas.pucp.edu.pe/index.php/educacion/article/view/5474

Coll, C., Monereo, C. (Ed.). (2008). Psicología de la educación virtual: aprender y enseñar con las tecnologías de la información y la comunicación. Madrid: Ediciones Morata.

Curci, R. (2003). Diagnóstico de la educación superior virtual en Venezuela. Caracas: Instituto Internacional para la Educación Superior en América latina y El Caribe. https://biblioteca.marco.edu.mx/files/01mtic_educacionvirtual_alc.pdf\#page=403

Gagliardi, V. (2020). Desafíos educativos en tiempos de pandemia. Question/Cuestión, 1(1), pp. 1-6. doi: https://doi.org/10.24215/16696581e312

Gros, B., \& Silva, J. (2005). La formación del profesorado como docentes en los espacios vir- tuales de aprendizaje. Revista iberoamericana de educación, 36(1), 1-13. doi: https://doi.org/10.35362/rie3612831 http://www.isiberico.com/documentos/tutorestudianteynuevorol.PDF La Madriz, J. (2016). Factores que promueven la deserción del aula virtual. Orbis. Revista 
Cien- tífica Ciencias Humanas, 12(35), 18-40. Recuperado de http://ojs.revistaorbis.org.ve/index.php/orbis/article/view/31

Llopiz, K., Andreu, N., Gónzalez, R., Alberca, N., Fuster-Guillén, D y Palacios-Garay, J. (2020). Prácticas educativas inclusivas a través de la educación a distancia.

Experiencias en Cuba. Propósitos y Representaciones, 8(2), 446. Recuperado de http://revistas.usil.edu.pe/index.php/pyr/article/view/446/971

Novoa Palacios, A. y Pirela Morillo, J. (2020). Acompañamiento desde una ética de la vida:

Para educar en tiempos de pandemia. Revista Internacional de Filosofía y Teoría

Social, 25(4), 11-24. doi: http://doi.org/10.5281/zenodo.3931040

Rugeles, P., Mora, B. y Metaute, P. (2015). El rol del estudiante en los ambientes educativos mediados por las TIC. Revista Lasallista de investigación, 12(2), 132-138. Recuperado de https://www.redalyc.org/pdf/695/69542291025.pdf

Silva, J. (2010). El rol del tutor en los entornos virtuales de aprendizaje. Innovación educativa, 10(52), $13-23$. Recuperado de https://www.redalyc.org/pdf/1794/179420763002.pdf

Thomas, G. y Loxley, A. (2007). Deconstrucción de la educación especial y construcción de la inclusiva. Editorial La Muralla. 